\title{
Gigagauss Magnetic Field Generation from High Intensity Laser Solid Interactions
}

\author{
J. Sefcik, M. D. Perry, B. F. Lasinski, A. B. Langdon, T. Cowan, J. Hammer, \\ S. Hatchett, A. Hunt, M. H. Key, M. Moran, D. Pennington, \\ R. Snavely, J. Trebes, S. C. Wilks
}

This paper was prepared for submittal to the

8th International Conference on Megagauss Magnetic Field Generation and Related Topics Talahassee, FL

October $18-23,1998$

October 15, 1998

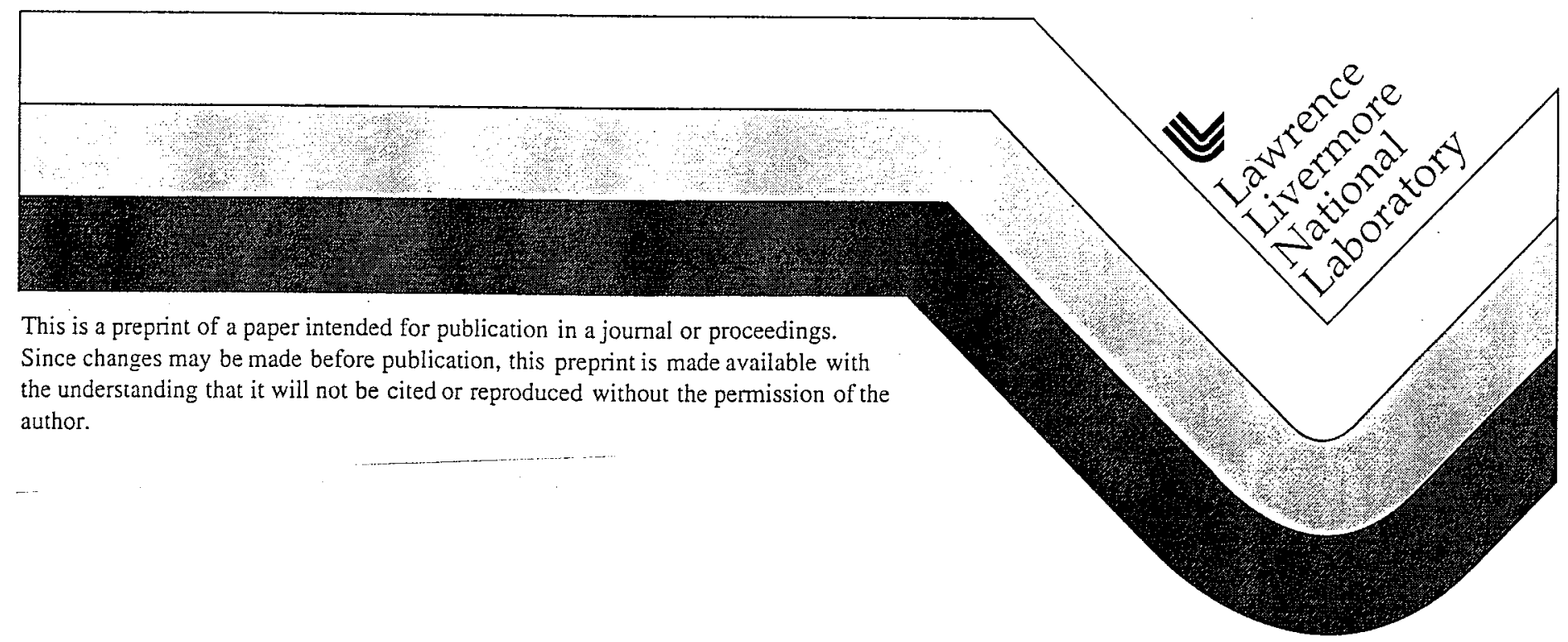




\section{DISCLAIMER}

This document was prepared as an account of work sponsored by an agency of the United States Government. Neither the United States Government nor the University of California nor any of their employees, makes any warranty, express or implied, or assumes any legal liability or responsibility for the accuracy, completeness, or usefulness of any information, apparatus, product, or process

disclosed, or represents that its use would not infringe privately owned rights. Reference herein to any specific commercial product, process, or service by trade name, trademark, manufacturer, or otherwise, does not necessarily constitute or imply its endorsement, recommendation, or favoring by the United States Government or the University of California. The views and opinions of authors expressed herein do not necessarily state or reflect those of the United States Government or the University of California, and shall not be used for advertising or product endorsement purposes. 


\title{
Gigagauss Magnetic Field Generation from High Intensity Laser Solid Interactions
}

\author{
J. Sefcik, M. D. Perry, B. F. Lasinski, \\ A. B. Langdon, T. Cowan, J. Hammer, \\ S. Hatchett, A. Hunt, M. H. Key, \\ M. Moran, D. Pennington, \\ $R$. Snavely, J. Trebes, and S. C. Wilks \\ Lawrence Livermore National Laboratory, Livermore, CA
}

\begin{abstract}
Intense laser $\left(>10^{21} \mathrm{~W} / \mathrm{cm} 2\right)$ sources using pulse compression techniques in the sub-picosecond time frame have been used to create dynamic electric field strengths in excess of $100 \mathrm{Megavolts} / \mathrm{mic}$ in with associated magnetic field strengths in the Gigagauss regime. We have begun a series of experiments using the Petawatt Laser system at LLNL to determine the potential of these sources for a variety of applications. Hot electron spectra from laser-target interactions in Au have been measured with energies up to $100 \mathrm{MeV}$. Hot $\mathrm{x}$-ray production has been measured using filtered thermoluminescent dosimeters and threshold nuclear activation $(\gamma, n)$ from giant resonance interactions. High resolution radiographs through a $\rho r \geq 165 \mathrm{gm} / \mathrm{cm}^{2}$ have been obtained. Dose levels in the $\mathrm{x}$-ray band from 2-8 MeV have been measured at the level of several Rads at one meter from the target for a single pulse. The physics of these sources and the scaling relationships and laser technology required to provide high magnetic fields will be discussed. Results of preliminary magnetic field calculations will be presented along with potential applications of this technology and estimates of the fundamental scaling limits for future development.
\end{abstract}

\section{Introduction}

The use of Petawatt class lasers for the production of copious amounts of high energy $x$ rays has been discussed for over a decade. ${ }^{1.2}$ Intermediate energy (0.1-1 MeV) $\mathrm{x}$-ray production with table-top size terawatt class lasers is now a subfield within the strong field interaction community. Numerous groups have observed electrons or bremsstrahlung spectra extending beyond $1 \mathrm{MeV}$.,.,+ 5.6

Recent experiments on the Petawatt laser at the NOVA laser facility at LLNL have demonstrated the short ( $<1 \mathrm{ps})$ laser pulses, when properly focussed on high-z targets, can produce hard, intense x-ray spectra that can be used for radiography. The outputs measured on these experiments imply very high electron current densities and current driven magnetic field strengths on the order of a gigagauss in extremely small volumes. 


\section{Experiments}

Petawatt shots for the production of high energy $x$-rays for radiography have been performed at the Lawrence Livermore National Laboratory's NOVA laser facility since September 1997. Typical laser data implies a pulse duration of $460 \pm 40$ fs and a compressor throughput of $84 \%$. Typical laser energy neasured before compression was $450 \mathrm{~J}$ in a $46.3 \mathrm{~cm}$ diameter beam. The laser beam and diagnostic configuration for the shots is shown in Figure 1. Due to the hole in the paraboloid, $7.4 \%$ of the incident energy is lost. The beam was focused using a Cassegrain telescope. The primary mirror was a paraboloid $(f=180 \mathrm{~cm})$ overcoated with an $\mathrm{HfO}_{2} / \mathrm{SiO}_{2}$ multilayer designed for high reflectivity at normal incidence. The secondary mirror was a $5 \mathrm{~cm}$ diameter fused silica flat which was overcoated with a $\mathrm{HfO}_{2} / \mathrm{SiO}_{2}$ multilayer high reflective coating for $1054 \mathrm{~nm}$ and placed $10 \mathrm{~cm}$ from the target. The beam size on the secondary mirror is $2.6 \mathrm{~cm}$ corresponding to an irradiance of $1.67 \times 10^{14}$ $\mathrm{W} / \mathrm{cm}^{2}$ for a nominal $400 \mathrm{~J}$ pulse incident on the mirror. At this irradiance, the top $\mathrm{SiO}_{2}$ layer is converted to a critical density plasma within the first 50 fs of the pulse. As a result, the bulk of the laser pulse is reflected not by the multilayer structure of the mirror but rather by the sharp gradient plasma surface. Measurements of the reflectivity from this plasma surface suggest a reflectivity of greater than $85 \%$ for the Petawatt puise.

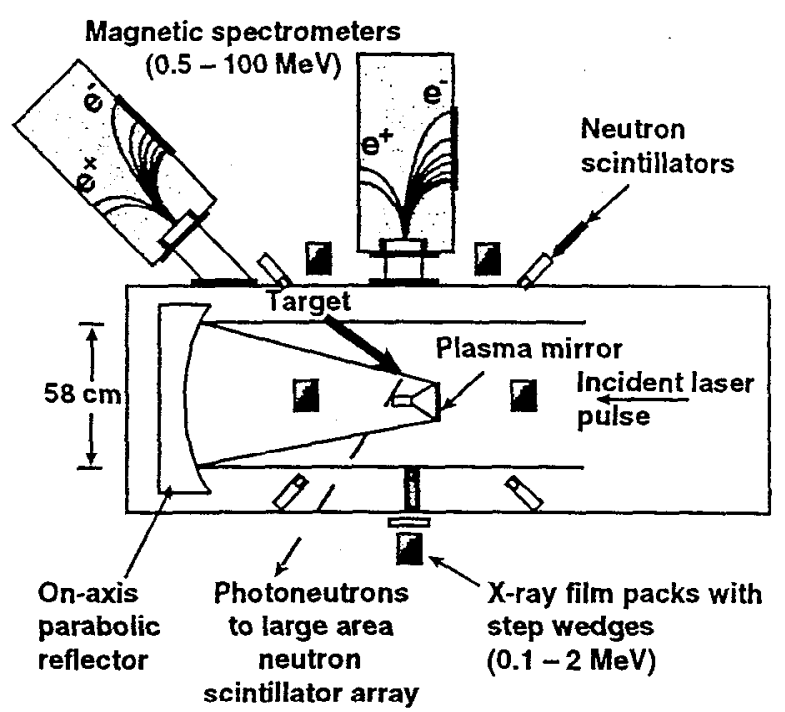

Figure 1. Diagnostic layout for Petawatt radiography experiments.

A typical target is shown in Figure 2. The laser beam strikes the surface of the gold target at near normal $\left(5^{\circ}\right)$ incidence producing a strong relativistic electron current with a complicated angular distribution into the target. Electrons greater than approximately $2 \mathrm{MeV}$ can escape the large space potential created 
within the target and penetrate the aluminum and $\mathrm{CD}_{2}$ backing material. Electron spectrometers are placed in the plane of incidence at $30^{\circ}$ from the laser axis and $95^{\circ}$ from the axis. These electron spectrometers consist of a permanent dipole magnet with emulsion as the detector. The total electron energy range covered in the spectrometer is $0.2-130 \mathrm{MeV}$, and the positron range is $0.2-40 \mathrm{MeV}$. The emulsion tracks are analyzed by the NASA/Marshall Space Flight Center group at Huntsville, Alabama.

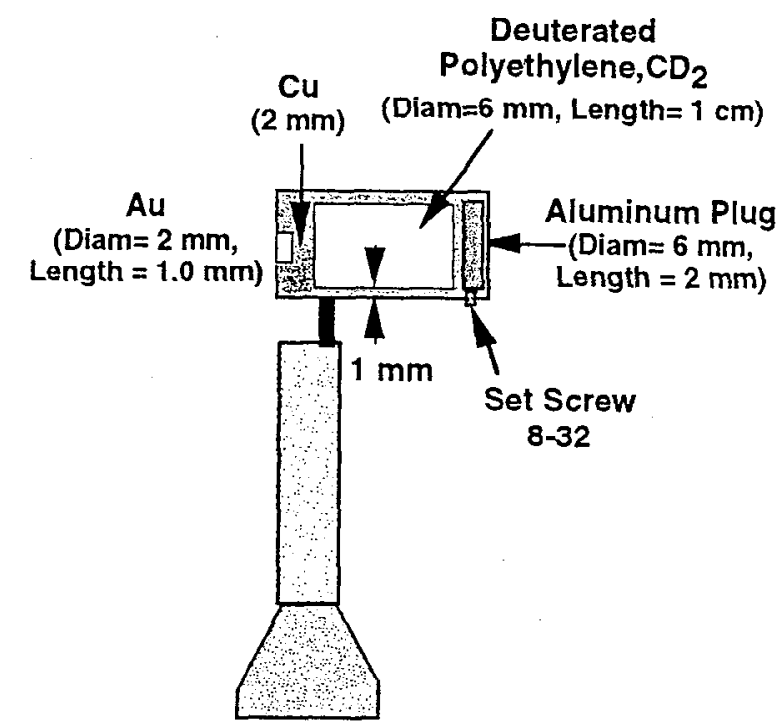

Figure 2. Petawatt radiography target.

An electron spectrum from a typical shot is shown in Figure 3. This shot used a gold target of $0.5 \mathrm{~mm}$ thickness. Electrons were observed at energies extending abovc $90 \mathrm{McV}$, however the bulk of the distribution was in the range of $-2-15 \mathrm{MeV}$ where the emission was found to be forward directed with about eight timcs more flux observed at $30^{\circ}$, with respect to the laser propagation direction, as compared to the flux at $95^{\circ}$. Note that an integration of the electron spectrum convolved with the bremsstrahlung cross section does not give an accurate estimate of the total photon yield or laser coupling efficiency since this electron spectrum is effected by the large space charge potential in the target and the energy loss $(d E / d x)$ through the target assembly. The energy loss through the target for the electrons depicted in Figure 3 was approximately $2.5 \pm 0.5 \mathrm{MeV}$. 


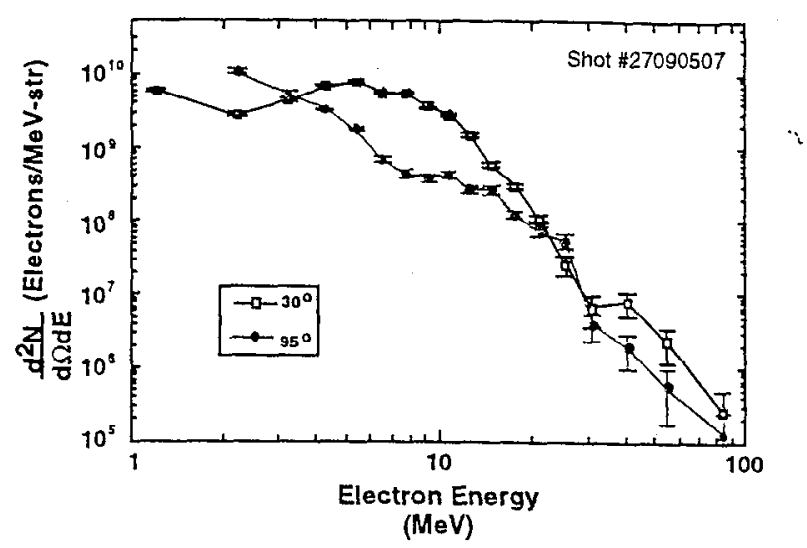

Figure 3. Electron spectra acquired at $30^{\circ}$ and $95^{\circ}$ with respect to the laser axis.

Aside from the suppressed region below $\approx 5 \mathrm{MeV}$, the elcctron spectrum can be well fit by a "two temperature" distribution. The spectrum observed at 30 degrees to the laser is shown in Figure 4 along with two quasi-Maxwellian distributions given by,

$$
N(E) d E=C\left(E^{1 / 2} / E_{e}^{3 / 2}\right) \exp \left[-E / E_{e}\right] d E,
$$

where $C$ is the scaling parameter and, $E_{e}$ is the characteristic electron energy. Detailed particle in cell (PIC) simulations suggest that this characteristic energy is well approximated by the cycle averaged quiver energy of a free electron within the intense field,

$$
\left\langle E_{c}\right\rangle=m_{e} c^{2}\left(1+2 U_{p} / m_{e} c^{2}\right]^{1 / 2},
$$

where $U_{p}(\mathrm{eV})=9.33 \times 10^{-14} I\left(\mathrm{~W} / \mathrm{cm}^{2}\right) \lambda^{2}(\mu \mathrm{m})$. The laser conditions for the spectrum depicted in Figure 4 were such that $\approx 50 \%$ of the $300 \mathrm{~J}$ incident on the target were focused within a $28 \times 40 \mu \mathrm{m}$ spot. This corresponds to an average irradiance of $8 \times 10^{19} \mathrm{~W} / \mathrm{cm}^{2}$ for this shot. The average quiver energy for this irradiance [from Equation (2)] is $2.95 \mathrm{MeV}$ in agreement with the characteristic electron energy for the bulk of the distribution of Figure 4. The incident laser spot also contained substructure which would have seeded self-focusing in the preformed plasma. This self-focusing is almost certainly responsible for the "hot tail" of the distribution characterized by a second Maxwellian with a characteristic electron energy of $11.5 \mathrm{MeV}$. This is consistent with PIC simulations which show the incident beam undergoing filamentation in the preformed plasma.

High-energy bremsstrahlung $x$ rays generated by these electrons in the gold target produced substantial radioactivity in the target assembly following the 
laser shot. This activity was from photonuclear reactions in both the gold and surrounding copper target-holder producing transmutation to platinum and nickel daughter isotopes. Positron emission was determined by observation of $511 \mathrm{keV}$ rrays resulting from annihilation radiation in the target assembly. Positive identification of both copper radioisotopes was established by fitting the decay curve with a two component decay with half-lives of $9.7 \mathrm{~min}$ and 12.7 min. The fit gives an identification probability of better than $99 \%$ for ${ }^{62} \mathrm{Cu}$ and ${ }^{64} \mathrm{Cu}$, respectively. The threshold gamma-ray energy for photo-activation of the gold is $8.06 \mathrm{MeV},{ }^{65} \mathrm{Cu}$ is $9.91 \mathrm{MeV}$ and $10.85 \mathrm{MeV}$ for ${ }^{63} \mathrm{Cu}$, indicating a large flux of high-energy bremsstrahlung.

High quality images of the far-field laser beam distribution are acquired on these shots. Beam distortion resulting from both pump-induced and thermal distortion in the Nova disk amplifiers has been corrected by the use of a deformable mirror. The system now can typically produce a well focused laser spot that is $9 \mu$ in diameter (FWHM). As a result, in the absence of self focusing, about $20 \%$ of the beam energy exceeds $=10^{20} \mathrm{~W} / \mathrm{cm}^{2}$. This suggests that most of the radiation generation is being produced by about 80 joules of the light incident on the target. Only the central spot would have had sufficient irradiance to produce the high energy electrons necessary to produce the hard $(>1 \mathrm{MeV}) \mathrm{x}$ ray photons.

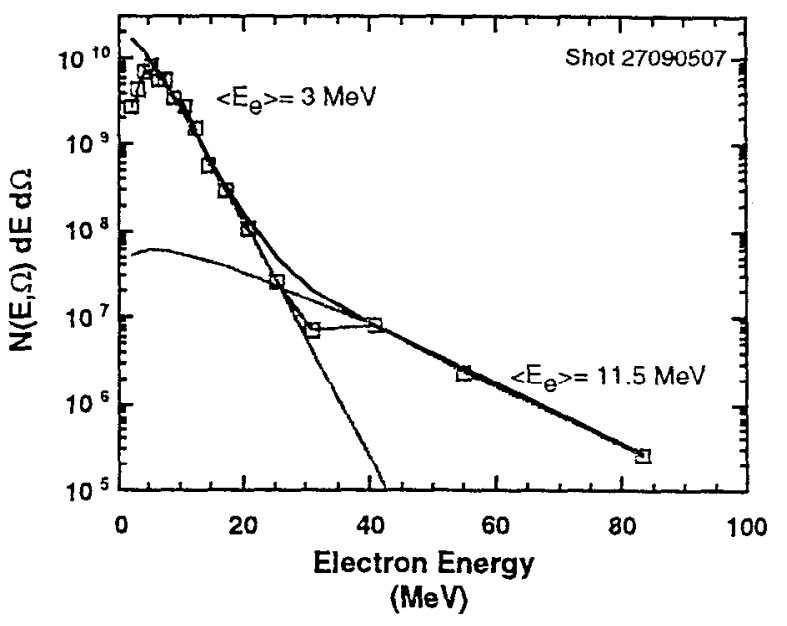

Figure 4. Electron spectrum and calculated Maxwellian distributions [Equation (2)]. The solid black curve is the sum of the two distributions.

Multiple radiographs thru varying thicknesses of lead are routinely acquired during shots. The resolution of the radiographs suggests that the spot size for high energy photon production from bremsstrahlung is less than 100 microns in diameter. These radiographs typically correspond to doses of $>1 \mathrm{MeV}$ photons as measured on thermoluminescent dosimeters of 0.1-2.0 Rads, depending on the parameters of the laser target interaction. Although the laser interaction is 
over within a few hundred femtoseconds, the electron beam produced in the target takes one or two picoseconds to complete its evolution and generate $\mathrm{x}$ rays from bremsstrahlung interactions.

\section{Calculations}

Calculations of conditions within the electron beam generation region have been performed using the 2-D particle-in-cell code ZOHAR. The model provides a fully kinetic and non-linear description of the laser-plasma interaction that incorporates Ampere-Faraday-Maxwell fields, relativistic particle dynamics, and self-consistent particle-in-cell coupling to the field equations. These calculations have been performed for laser hole-boring in plasma slabs with electron densities $\left(n_{e}\right)$ that are 5-50 times the critical density $\left(n_{c}\right)$. Individual particle orbits are tracked to study the evolution of the static magnetic and electric fields produced by the laser driven electrons.

A typical plot from a ZOHAR calculation of a $I=10^{21} \mathrm{~W} / \mathrm{cm}^{2}$ laser pulse of $1 \mu$ light interacting with a plasma slab at $n_{e}=50 n_{c}$ is shown in Figure 5. These laser intensities are somewhat higher than those currently being achieved experimentally. The highest intensity magnetic fields are shown in this plot to exceed 500 megagauss over a region of approximately $50 \mu^{3}$. Fields in excess of 100 megagauss span a region approximately $1000 \mu^{3}$. Peak fields in this calculation reached approximately 1.5 gigagauss. Analysis of a number of calculations at laser intensities between $10^{20} \mathrm{~W} / \mathrm{cm}^{2}$ and $10^{21} \mathrm{~W} / \mathrm{cm}^{2}$ suggest that the static magnetic field intensity generated by the electron beam formed by the laser reaches peak levels that are approximately $1 / 3$ the dynamic magnetic field level in the incident laser beam. The duration of the magnetic field is on the order of picoseconds.

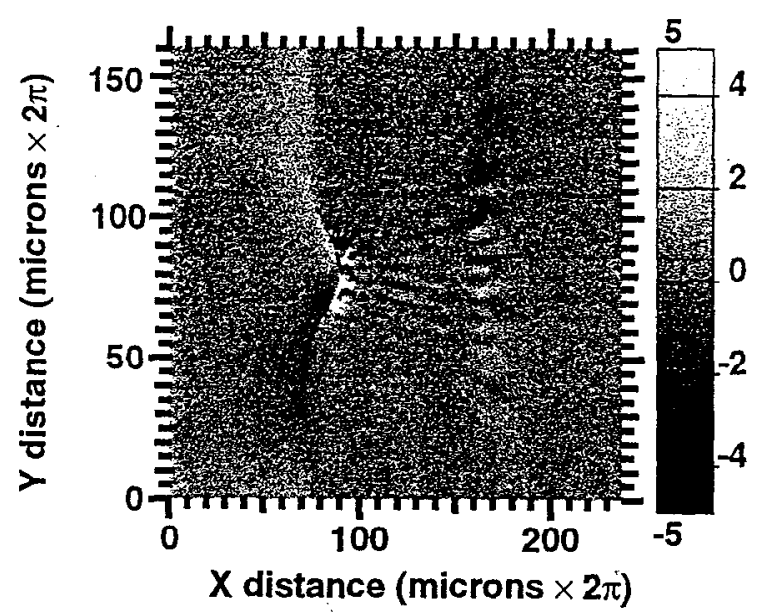

Figure 5. Magnetic field intensity produced by laser-plasma interaction. The vertical intensity scale is in units of 100 megagauss and is capped at 500 megagauss. 


\section{Future Work}

Experiments with the Petawatt laser at the NOVA facility will continue to examine the optimization of the electron beam generated by the laser-plasma interaction for production of suitable $x$-ray sources for radiographic applications. Laser improvements will be incorporated to increase the fraction of the laser beam that is above the required intensity for the production of hot electrons. Grating development is underway to produce dielectric transmission gratings that will be capable of handling a $5 \mathrm{~kJ}$ pulse. Such a pulse, properly formatted, would increase the electron current by a factor of 50 over the present experiments. Further improvements using tailored target design might be possible. Measurements of the magnetic field intensity near the laser-plasma interaction region may be attempted.

\section{Acknowledgement}

This work was performed under the auspices of the Department of Energy by the Lawrence Livermore National Laboratory under Contract W-7405-Eng48.

\section{References}

1. W. Priedhorsky, D. Lier, R. Day, and D. Gerke, "Hard x-ray measurements of 10.6 micron Laser Irradiated Targets," Physical Review Letters, 47, 1661 (1981).

2. M. D. Perry, C. Keane, and E. M. Campbell, "Ultrahigh Brightness Lasers and their Applications," LLNL Internal document (1987).

3. J. D. Kmetec, C. L. Gordon, J. J. Macklin, B. E. Lemoff, and S. E. Harris, "MeV X-ray Generation with Femtosecond Lasers," Physical Review Letters, 68, 1527 (1992).

4. G. Malka and J. L. Miquel, "Experimental Confirmation of PonderomotiveForce Electrons Produced by an Ultrarelativistic Laser Pulse on a Solid Target," Physical Revicw Letters, 77, 75 (1996).

5. G. Malka, E. Lefebvre, J. L. Miquel, and C. Rousseaux, "Observation of Relativistic Electrons Produced in the Interaction of 400 Fsec Laser Pulses with Solid Targets," Anomalous Absorption Conference, Fairbanks, Alaska, August 1996.

6. P. Gibbon, "Efficient Production of Fast Electrons from Femtosecond Laser Interaction with Solid Targets," Physical Review Letters, 73, 664 (1994). 